\section{THE PRINCIPLES OF TREATMENT IN} GASTROPTOSIS.*

BY

ROBERT HUTCHISON, M.D., F.R.C.P., PHYSICIAN TO THE LONDON HOSPITAL.

I PROPOSE to deal only with the general principles which should guide one in the treatment of gastroptosis, for, if these are agreed to, the details will follow as a matter of course.

The first point upon which it is necessary to lay emphasis is that gastroptosis is not really in itself a disease, and in many cases calls for no special treatment. It must have happened to every one present to discover the existence of displacement of the stomach in the course of the routine examination of a patient who has made no complaint of gastric symptoms. It will be found, I think, in such cases that the dislocation of the stomach has been indpopd, so to speak, accidentally-most often, perhaps, as a result of weakening of the abdominal wall in consequence of repeated child-bearing. It is true that in a case of this sort there may be a greater or less degree of "pendulous belly" which may cause some mechanical inoonvenience; but, apart from that, and assuming the patient to be otherwise vigorous, there need be no symptoms which can be directly referred to the abdominal organs.

I have said that in these oases gastroptosis demands no spocial treatment, but I believe I might go further and state that in no circumstances does mere displacement of the stomach, considered by itself, make such demands, and that it is only because of its tendency to be associated with two other morbid conditions that gastroptosis comes within the cognizance of the physician at all. These morbid conditions are (1) a general state of neurasthenia and (2) functional disorder of the stomach and intestines A word or two must be said about the relation to gastroptosis of each of these common concomitants.

Relation of Neurasthenia to Gastroptosis.

Every one will allow that in many, perhaps most, cases of gastroptosis the patient exhibits the general symptoms and signs of neurasthenia, but as to whether the gastroptogis causes the neurasthenia or vice verse, there is by no means the same measure of agreement.

According to some, displacement of the stomach is merely one of the stigmata of neurasthenia-the outward and visible sign of an inward constitutional state. Stiller is, perhaps, the chief exponent of this view, and he has labelled the constitutional state referred to by the special name of "morbus asthenicus" and has given an elaborate description of the features by which it can be recognized.

In the view of others displacement of the stomach is itself a cause - and, indeed, in the eyes of some enthusiasts the chief cause- of neurasthenia. There is perhaps some trath in both views, and the gastroptotic patient may be one condemned to revolve in a vicious circle-the displacement of his stomach favouring (in ways immediately to be described) functional dyspepsia, and the dyspepsia leading to impairment of nutrition and nervous exhaus. tion, which in its turn perpetuates the dyspepsia, and lessens the tone of the abdominal muscles which should support the stomach. We have thus established a chain of cause and effect, which proceeds on the lines of the fable which describes the House that Jack Built. Be this as it may, there can be no doubt that in the majority of cases the treatment of gastroptosis resolves itself largely into the treatment of a condition of neurasthenia.

Relation of Functional Dyspepsia to Gastroptosis.

Just as patients who exhibit gastroptosis usually show also the signs of nervous exhaustion, so are they prone to suffer in addition from functional disorders of the stomach. Bat we have already seen that the mere displacement of the stomach does not by itself necessarily interfere with the normal discharge of its functions, and hence the conclusion seems justified that the dyspepsia is in the main the result of the condition of the nervous

* Read before the Medical Society of London. system. This need not surprise us, for every one knows how prone neurasthenia is to result in gastric derangement even when the stomach occupies its normal situation. As has been hinted above, however, it is possible that this does not cover the whole truth of the matter. It is at least possible that the fact of the stomach being out of place favours, if it does not actually cause, some interference with the performance of its functions. It is unlikely that this should be the case as regards disorders of secretion, but it is at least conceivable that displacement offers some mechanical hindrance to the due performance of the function of motility. Various modes in which this may happen have even been specified, such as the occurrence of a hypothetical kinking at the pyloroduodenal junction, or one is told that there must be a difficulty in the organ emptying itself uphill. For my own part, I do not attach much weight to these imaginings, and am inclined to agree with those who would attribute the disorder of function met with in gastroptosis to the accompanying neurasthenic state rather than to the mere fact of displacement. At all events it is quite certain that functional dyspepsia is a common concomitant of gastroptosis, and may call urgently for treatment, and it therefore becomes of importance to state in rather more precise terms what forms the functional disorder usually assumes.

On this point, fortunately, it is possible to speak with some degree of confidence, and one may say without hesitation that the functional disorders most commonly associated with gastroptosis are disturbance of motility on the one hand and of secretion on the other, and, further, that both disorders often coexist.

The disturbance of motility takes the form of impairment of gastric " tone " and an enfeeblement of expulsire power. It is, perhaps, advisable to state rather more precisely what one means by "tone." I imply by it the power of the stomach to "grasp" its contents in such a way as to maintain the intragastric tension at a constant level. The mechanism by which this is brought about is not fully understood, but I believe that it is usually impaired in cases of gastroptosis. Hence the occurrence of splashing. The diminution of expulsive power is shown by some degree of delay in the onward passage of the contents of the organ, but it is noteworthy that careful observations have shown that this never amounts to actual stagnation. ${ }^{2}$ The bearing of this on treatment will be seen later.

The disturbance of secretion usually takes the form of an over-production of acid; more rarely one meets. with hypochylia, or even complete achylia.

Although these are the chief forms of functional defect met with, it must be noted that a displaced stomach is apt also to be hyperaesthetic, and, further, that the functional defects may be accompanied by, or even lead to, actual organic disease, of which chronic gastritis is the chief.

\section{Treatment}

From these preliminary observations it will be seen that the treatment of gastroptosis is a complicated matter, and resolves itself usually into three branches : first, and of most importance, the treatment of a general condition of neurasthenia; secondly, the remedying of any functional disorder of the stomach which may bappen to be present; thirdly, and of least importance, the correction, so far as is possible, of the malposition of the organ.

In the remainder of this paper I propose to deal briefly with the principles to be observed in carrying out each of these lines of treatment.

\section{The Treatment of the Neurasthenic State.}

In the treatment of the neurasthenia, which accompanies if it does not underlie most cases of gastroptosis, our most effective means is a well-planned rest cure. Our. object must be to improve nutrition, or, in the words of Weir Mitchell, to make fat and blood. At the outset one. a priori objection to this plan must be faced. To improve nutrition the patient must be overfed; bat will his debilitated stomach permit of this being done? Is there. not a danger of over-distending it and of increasing any atonic dilatation which may be present? In practioe I do not think this objection holds. Provided the feeds be not too large and the food suitably selected and prepared, such a result need not be feared; and as the general 
"tone" of the patient improves one finds that the digestive power improves pari passu. Furthermore, one finds that patients with gastroptosis who suffer great digestive discomfort whilst they are going about lose most of their symptoms so soon as the recumbent position is adopted, and have no difficulty in managing a fall diet. Per contra there is, I am sure, a real danger in cut. ting down the diet in these cases in order to quiet the complaints of the stomach. By so doing one merely suppresses a symptom whilst really aggravating the com. plaint, for the consequence of such a course is that the nutrition becomes still further impaired and the neurasthenic state exaggerated. Here, as always, in pre. scribing dietetic rules one must not sacrifice the whole body for the sake of any one member of it, or, in other words, one must diet the patient and not the disease.

Of the accessory measures in the rest cure-massage and electricity-most importance, I think, is to be attached to massage. It has a threefold use. It promotes general nutrition and the laying on of fat; it improves gastric and intestinal tone, and it strengthens the abdominal wall-all of which are cardinal objects of treatment. The help of electricity can, in my judgement; be dispensed with, but to this point I shall return again.

Concerning the use of drugs in combating the neur. asthenio state there is little to say; the bromides are perhaps the most generally useful, and will usually be called for at some period in the case. Strychnine, though often ordered, is frequently injurious. Of such agents as the hypophosphites and glycerophosphates I have a low opinion. Iron may be helpful if there is pronounced anaemia, but that is an exceptional occurrence, and the state of the digestion will not always permit of its exhibition. In such cases arsenic may come to our assistance.

\section{The Treatment of any Functional Disorder of the} Stomach which may be Present.

I have put the treatment of the condition of "asthenia" first in describing the management of gastroptosis because I believe it to be far the most important. To be successful one must conoentrate one's attention on the patient and not on his stomach, but if, incidentally, one can do anything to relieve any disorder of gastric function which may be present, so much the better. We have seen that these may be of three sorts: (1) Impairment of motility and tone, (2) disorder of secretion, (3) increase of sensibility.

A word or two will suffice to indicate the principles to. be observed in the treatment of each of these disorders.

In ameliorating the general asthenic condition of the patient by a rest cure we are already doing much to correct any loss of tone or motility in the stomach. This has been already indicated, so I need not stop to labour the point. There is experimental evidence also that massage of the stomach stimulates its movements and so aids its motility. As to the value of electrical means, I am not convinced. I believe that there is no satisfactory evidence that the application of electricity to the abdominal surface has any effect upon the stomach at all but if any form of it is to be tried the sinusoidal current is probably that which offers the best chance of success. Drugs also, contrary to what might be expected, are probably of little help. We stand much in need of some drug which will act upon the musculature of the stomach in the same specific way as digitalis does upon that of the heart or ergot upon that of the uterus. Physostigmine is perhaps the one from which, on pharmacological grounds, most might be expected in this direction, but its use is open to practioal objections, and it has never been generally adopted. Strychnine may possibly increase the tone of the stomach as it does that of most unstriped muscle, but the results it yields are not brilliant, and its action apon the nervous system is sometimes disadvantageous. I am not sure that aloes has not a stimulating effect upon the morements of the stomach, as it has upon those of the colon. I have an impression that it has, but it is difficult to adduce any convincing clinical evidence of such action, and I am not aware whether it has been tested experimentally.

The means to be adopted in the correction of disorders of secretion in cases of gastroptosis differ in no respect from those in use in ordinary cases of functional dyspepsia.
Orersecretion is, as has been pointed out, the commonest fault, and is to be met by the free administration of fats and by the judicious use of bismuth and the earthy carbonates. The bromides are also of service here, as they seem to deaden the reflex excitability of the stomach.

In the rarer esses in which diminished secretion or even complete achylia is met with it may be necessary to linit the amount of meat in the diet, or, at all events, to present it only in a finely divided form, and some help may be obtained from the use of artificial substitutes for the gastric juice, such as hydrochloric acid or acidol. The administration of pepsin is, however, in my opinion, of doubtful utility.

In the treatment of hyperaesthesia of the displaced stomach there is no measure, I believe, egual in vilue to the use of local moist heat in the form of poultices or fomentations, and whenever there is general epigastric tenderness such means may be usefally employed during the first week of the rest cure. Gastric sedatives, such as bismuth, bromides, chloroform, and even, in extreme cases, opium, are also of service here. Should the disorder of function have induced or be complicated by some degree of gastritis, as evidenced by a foul tongue, impairment of appetite, and nausea, it is advisable, before treating the underlying disorder of secretion or motility to allay the catarrhal symptoms. The diet in such circumstantes should consist of milk only, and judicious use should be made of those well-tried drugg-mercury and rhubairb.

I have said nothing so far of the treatment of the disorders of intestinal function which may complicate a cose of gastroptosis, but a few words on this important matter may not be out of place. Constipation is the condition with which in the great majority of cases one has to deal. This is due to several factors. In part it is the consequence of the spare diet on which the patient has usually been living when he comes under treatment; in part it is no doubt due to diminished power of peristalsis, but in a large degree it is not a true constipation, but what Dr. Hertz calls a condition of "dyschezia," or difficult defaecation, the result of a defective power of expulsion which the enfeebled abdominal wall entails.

So soon as the patient is receiving a full diet the first of these factors is abolished; abdominal massage contributes to overcoming the second, but the help of "tonic" aperients may be necessary in addition-at the outset, at all events. The factor of dyschezia is met by strengthening the abdominal wall by the methods shortly to be considered, but in the early stages of treatment the help of enemata may also be required as the most effectual and rational means of overcoming it.

The Displacement of the Stomach.

I have left to the last the consideration of the means to be employed for replacing the stomach in its natural position, because I believe this, for reasons already given, to be a matter of secondary importance. Unquestionably patients with enteroptosis derive great comfort from any effective means of supporting the abdominal viscera, but it is very doubtful whether this is the result of any actual pushing up of the displaced organs. Such support gives relief from those feelings of sinking, emptiness, and exhaustion which are amongst the most bitter complaints of the gastroptotic, but these sensations are probably due to lessened intra-abdominal tension and a tendency to pooling of the blood in the splanchnic area when the erect position is assumed. The relief of these symptoms is, none the less, a legitimate object of treatment, and hence the provision of some form of abdominal support has always to be considered. Now it has been truly remarked that the best belt is a firm abdominal wall, and hence our efforts should be directed to restoring the tone of the abdominal wall and to thickening it by the deposition of a substantial layer of subcutaneous and extraperitoneal fat. The rest cure, if successful, should achieve the second of these objects, especially in men, in whom the deposition of fat in the abdomen seems to take place more easily than in women. By increasing the amount of "packing" in the abdominal cavity, intra-abdominal tension is increased attd the tendency to undue mobility of the viscera restrained. I doubt, however, whether the gain in abdominal adiposity really does much towards replacing a seriously dislocated stomach. Certainly I have known cases in which, in spite of a total gain in weight of nearly $20 \mathrm{lb}$. as the result 
of a rest cure, the stomach remained in practically the same position as before.

As regards the development of the abdominal muscles, reliance must be placed upon massage and the practice of "abdominal exercises." Electrical treatment may un. doubtedly be of help here, but its results are probably inferior in value to the spontaneous muscular contractions which exercises provoke. Hydrotherapeutic measuresfor example, the Scottish douche-are also of service in increasing the tone not only of the abdominal wall, but also, in all probability, that of the blood vessels of the splanchnic area as well. The question of artificial substitutes for the abdominal wall will always arise.

The argument sometimes used against them-especially by masseurs-that they demoralize the abdominal muscles by doing their work for them, is, I.believe, fallacious. On the contrary, by preventing over-stretching of the weakened abdominal wall, they are undoubtedly of service, pending the restoration of the natural means of support. They are therefore of great temporary utility, at least. It wonld be going too far into details to consider the relative merits of the different forms of artificial support which have been devised, but there is certainly no lack of choice-from the simple plaster strapping of Achilles Rose to the latest belt-corset of the artist in orthopaedic appliances. I thlnk it will be generally agreed, however, that the ideal abdominal belt remains yet to be invented. It is simple enough to point out what is wanted-the exercise of pressure from the lower part of the abdomen upwards and somewhat backwards, but the difficulty is to carry this principle into practice. In cases of "pendulous belly" in stout subjects it is quite simple, but most patients with gastroptosis who require treatment are, ex hypothesi, thin, with prominent iliac bones which tend to carry off the pressure from the hypogastric region where we most wish to apply it. The problem is a purely mechanical one, and the use of a suitable pad helps to overcome it, bat the results often leave much to be desired.

Having by the adoption of the above means restored the patient to a condition of comparative well-being, the next problem is how to keep him so. It is here that our real difficulties begin, for the neurasthenic state, which, as has been repeatedly pointed out, underlies the gastroptosis in the great majority of instances, is due either to an inborn feebleness of constitution or to unfavourable factors in the environment, of which the former is wholly and the latter often very largely beyond our power to alter. Much may be done, however, by suitable regulation of the patient's life and habits. Overstrain-mental, physical, and emotional-must be avoided so far as is possible. The importance of rest must be enjoined, particularly rest after meals, which greatly facilitates the labours of the stomach. Frequent holidays are advantageous. The diet must be so regulated as to contain a maximum of nutriment in a minimum of bulk, and all voluminous and innutritious foods forbidden. It is particularly important, as in all cases of gastric atony, that the meals should be dry. Meanwhile, the further development of the abdominal wall must be persevered with by the persistent practice of abdominal exercises, coupled, perhaps, with the use of anto-massage, and, until a sufficient degree of natural support bas been attained, the belt must not be left off.

The use of drugs may be confined to the occasional administration of bromides, or of such as are required for the relief of any particular symptoms. Suitable aperients may be necessary to supplement the use of enemata.

In those cases in which the carrying out of a thorough rest cure is impossible, the above means may be used as best caiculated to palliate the symptoms, but it will be readily understood that a patient with gastroptosis whose symptoms have been sufficiently severe to call for treatment is rarely able to retarn to a condition of full actipity. He must walk softly and be sparing of his nervous output, as otherwise a relapse is almost certain to ensue. It would not be an exaggeration, indeed, to say. that his whole life must partake of the nature of a more or less modified rest-cure.

Up to this point we have been concerned with the things to be done in the treatment of gastroptosis; may I conclude by enumerating three things which I think should not be done? First, patients with gastroptosis should not be overdieted-which, of course, is just the reverse of being overfed. Secondly, their stomachs should not be washed out. Thirdly-and pace Mr. Eve - they should not be operated upon. They should not be overdieted, because if that is done their nutrition is apt to suffer, and the maintenance of a high degree of nutrition is the first essential of successful treatment. They should not be washed out, because there is no real gastric stagnation, and the introduction of large quantities of fluid is apt to overdistend the already atonic stomach. They should not be operated upon, because the mere replacement of the stomach, even if this be permanently possible by operation, is treating a symptom and not the real disease, and because most patients with gastroptosis are neurasthenic, and no operation on a neurasthenic subject is advisable without very good reason.

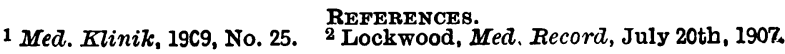

\section{A CASE OF CEREBRO-SPINAL RHINORRHOEA WITH DOUBLE OPTIC ATROPHY.}

BY NORMAN H. PIKE, M.B., B.S.LOND., SURGEON FOR THE EYE, FAR, NOSE, AND THROAT, THE GENERAT HOSPITAT, CHELTENHAM.

AN undoubted case of the spontaneous escape of cerebrospinal fluid from the nose, known as "cerebro-spinal rhinorrhoea," is of safficient interest to record.

A woman aged 22 first attended among my out-patients on her right nostril.

History.

Up to the age of 12 she was a strong, healthy girl, and could see well. From 12 to 13 she bad a severe illness, keeping her bed for nearly twelve months. The chief symptoms were severebea headache, vomiting, drowsiness, frequent convulsions in which she was unconscious, and stiffness at the back of the neck. The eyes becam

quite blind. After getting up she continued to have severe attacks of pain often nocturnal, and occurred about every three weeks to one month, but in the year 1908 they were less frequent-about. every three months. Mr. Arthur Cardew, who attended her during her illness, told me that there was some pyrexia for the first few days, but that afterwards the illness ran an apyrexial course.

\section{Family History.}

The only interesting fact about the family history that I have discovered is that her younger sister has suspiciously notched discovered is that her younger sister has suspiciously notched general expression, too, suggests congenital syphilis.

$$
\text { Present Condition. }
$$

She is a moderately well developed girl with a prominent head, measuring round the forehead $55 \frac{1}{\mathrm{~cm}} \mathrm{~cm}$. Mentally she is dull; her speech is slow and the voice has a monotonous character. She is quite blind and has no perception of light. There is no ocular paralysis, but marked nystagmoid movements of the eyeballs of an ocular character. The pupils areequal moty dilated, and react slightly to a strong light. equal, moderately dilated, and react slightly to a strong light. lamina cribrosa is indistinct and the vessels are tortuous, the condition being one of double post-neuritic atrophy.

The nose is one of double post-neuritic atrophy. anterior rhinoscopy nothing abnormal is seen, except that on anterior rhinoscopy nothing abnormal is seen, except that on turbingl and extending into the inferior meatus, there is an tarbinal and extending into the inferior mestus, there is an a constant dripping of a clear watery fluid. There is no discharge from the left nostril. With the patient in the Killian. position the fluid is seen to collect between the middle turbinal. and the septum, and the tumour has probably been formed by the continual soaking of the spongy tissues. There is no suppuration in the nose and no signs of any sinus trouble. Transillumination shows good infraorbital reflexes on both sides.

During the patient's attendances at the hospital the following facts were ascertained about this fluid:

acts were ascertained about this fluid : as in epistaxis.

2. It dropped at the rate of from 3 to 8 drops a minute, and was measured on several occasions, the amounts varying from $3 \mathrm{vj}$ to $3 \mathrm{j}$ in an hour and a half-that is, from 12 to $16 \mathrm{oz}$. in wenty-four hours. It drops faster with the head forward.

3. It was ascertained from the mother that when asleep she makes frequent swallowing movements, tho fluid apparently running into the naso-pharynx.

4. She uses many handkerchiefs a day, and these when dried. are found to be quite pliable and not at all stiff.

5. Cocaine and adrenalin applied had no effect on the flow.

6. The fluid has never ceased during her attendances, and has now been constant for over nine months. Since its advent the 